\title{
GENERALIZED PASCAL TRIANGLES: OVERVIEW OF NEW RESULTS
}

\author{
IVAN KOREC \\ Mathematical Institute, Slovak Academy of Sciences \\ Štefánikova 49,81473 Bratislava, Slovakia
}

1. Introduction and definitions. The present paper deals with an algebraical generalization of the Pascal triangle. In this sense it differs from most frequently studied various arithmetical generalizations; see e.g. Bondarenko's book [1] with many of more than 400 items in its bibliography. The classification is neither exclusive nor sharp; Pascal triangles modulo $n$ belong both to arithmetical generalizations and the algebraical generalization presented here. The immediate motivation for introducing the latter was the study of structure of real-time systolic trellis automata (see [4], [5], later also [7], [8], [9]). However, the algebraic generalization presented is also suitable for describing computations of one-dimensional cellular automata.

The previous overview was [13], where [10] and [11] were summarized. The present paper mainly summarizes [16] and [17]. [14] deals directly with a kind of systolic trellis automata. In [15] it is shown that Pascal triangle modulo $n$ is modular or strictly modular if and only if $n$ is a prime power or a prime, respectively. Modularity and strict modularity of trellises were introduced in [2] and [3].

Let $\mathbf{N}$ denote the set of nonnegative integers and for every $n \in \mathbf{N}$ let

$$
\mathbf{D}_{n}=\{(x, y) \in \mathbf{N} \times \mathbf{N}: x+y \geq n-1\} .
$$

The operators DIV, MOD will denote the quotient and the remainder by the integer division (like in the programming language PASCAL).

If $\mathbf{A}$ is an alphabet (i.e., a finite nonempty set) then $\mathbf{A}^{+}$will denote the set of all nonempty words in the alphabet $\mathbf{A}$. The length of a word $w$ will be denoted

1991 Mathematics Subject Classification: Primary 68Q80, 11B37; Secondary 68R15.

This work was supported by Grant 364/1991 of Slovak Academy of Sciences.

The paper is in final form and no version of it will be published elsewhere. 
by $|w|$; it must be distinguished from the absolute value of a real by the context. The $i$ th symbol of $w$ will be denoted by $w(i)$; the starting symbol is $w(0)$ and hence the last symbol is $w(|w|-1)$. Also some further usual notations from the theory of formal languages will be used.

By an algebra we shall always understand an algebra $\mathcal{A}=(\mathbf{A} ; *$, o $)$ of signature $(2,0)$ and satisfying the identity $\mathrm{o} * \mathrm{o}=\mathrm{o}$. We shall usually consider finite algebras; the exceptions will be explicitly mentioned.

Definition 1.1. With every algebra $\mathcal{A}=(\mathbf{A} ; *, \mathrm{o})$ and every $w \in \mathbf{A}^{+}$we associate the function $G=\operatorname{GPT}(\mathcal{A}, w)$ with the domain $\mathbf{D}_{|w|}$ by the formulae

$$
G(x, y)= \begin{cases}w(x) & \text { if } x+y=|w|-1, \\ 0 * G(0, y-1) & \text { if } x=0, y \geq|w|, \\ G(x-1,0) * \mathrm{o} & \text { if } y=0, x \geq|w|, \\ G(x-1, y) * G(x, y-1) & \text { if } x+y \geq|w|, x>0, y>0 .\end{cases}
$$

The functions of the form $\operatorname{GPT}(\mathcal{A}, w)$ for a finite algebra $\mathcal{A}$ and a word $w \in \mathbf{A}^{+}$ will be called generalized Pascal triangles (abbreviation: GPT).

When displaying a GPT we shall always assume the corresponding coordinate system in the plane: the axis $x$ is directed left-down and the axis $y$ is directed rightdown. Nice (and very often studied) examples of GPT are Pascal triangles modulo $n$, particularly if $n$ is a prime or a prime power. The value $G(x, y)=\left(\begin{array}{c}x+y \\ x\end{array}\right) \operatorname{MOD} n$ is always written into the unit square $\langle x, y\rangle$. We can express these GPT in the form $\operatorname{GPT}\left(\mathcal{N}_{n}, 1\right)$, where $\mathcal{N}_{n}=(\{0,1, \ldots, n-1\} ;+, 0)$ and + denotes the addition modulo $n$.

Definition 1.2. Let $\mathcal{A}=(\mathbf{A} ; *, \mathrm{o})$ be an algebra, $w \in \mathbf{A}^{+}$and $t \in \mathbf{N}$.

a) The $t$-th row of $G=\operatorname{GPT}(\mathcal{A}, w)$, notation: $\mathrm{R}(\mathcal{A}, w ; t)$, will be the word consisting of

$$
G(0, t+|w|-1), G(1, t+|w|-2), \ldots, G(t+|w|-2,1), G(t+|w|-1,0) .
$$

b) The substantial part $\operatorname{SP}(\mathcal{A}, w ; t)$ of the $t$ th row of $G=\operatorname{GPT}(\mathcal{A}, w)$ will be the empty word if $G(x, t-x)=\mathrm{o}$ for all $x \in \mathbf{Z}$; otherwise $\operatorname{SP}(\mathcal{A}, w ; t)$ will be the word consisting of

$$
G(u, t+|w|-u-1), G(u+1, t+|w|-u-2), \ldots, G(v, t+|w|-v-1),
$$

where $u$ is the least and $v$ is the greatest integer such that $G(u, t+|w|-u-1) \neq 0$ and $G(v, t+|w|-v-1) \neq$ o.

c) $\operatorname{lmarg}(\mathcal{A}, w ; t)$ and $\operatorname{rmarg}(\mathcal{A}, w ; t)$ will denote the number of (occurrences of) o in $\mathrm{R}(\mathcal{A}, w ; t)$ before and after $\operatorname{SP}(\mathcal{A}, w ; t)$, respectively; if $\operatorname{SP}(\mathcal{A}, w ; t)$ is empty then $\operatorname{lmarg}(\mathcal{A}, w ; t)=|w|+t$ and $\operatorname{rmarg}(\mathcal{A}, w ; t)=0$.

In other words, $\operatorname{SP}(\mathcal{A}, w ; t)$ is the least (connected) subword of the $t$ th row of $\operatorname{GPT}(\mathcal{A}, w)$ which contains all its symbols distinct from o. Further, always

$$
\mathrm{R}(\mathcal{A}, w ; t)=\mathrm{o}^{\operatorname{lmarg}(\mathcal{A}, w ; t)} \mathrm{SP}(\mathcal{A}, w ; t) \mathrm{o}^{\operatorname{rmarg}(\mathcal{A}, w ; t)} .
$$


Remark 1.3. Originally (for example, in [11] or [13]), generalized Pascal triangles were associated with the algebras of signature $(0,1,1,2)$ (but the approach introduced above was also mentioned). The algebra corresponding now to the original algebra $\mathcal{B}=(\mathbf{B} ; K, \mathrm{l}, \mathrm{r},$.$) can be \mathcal{A}=(\mathbf{A} ; *, \mathrm{o})$, where $\mathbf{A}=\mathbf{B} \cup\{\mathrm{o}\}$, $\mathrm{o} \notin \mathbf{B}$ and

$$
x * y=x . y, \quad x * \mathrm{o}=\mathrm{r}(x), \quad \mathrm{o} * y=\mathrm{l}(y), \quad \mathrm{o} * \mathrm{o}=\mathrm{o}
$$

for all $x, y \in \mathbf{B}$. The constant $K$ (which was used to define $\operatorname{GPT}(\mathcal{B})=\operatorname{GPT}(\mathcal{B}, K)$ ) has no analogy here; therefore $\operatorname{GPT}(\mathcal{A})$ is not defined now. Notice also that sometimes it is more suitable to choose o $\in \mathbf{B}$; e.g. if we consider the Pascal triangle modulo a positive integer $n$, the choice $\mathrm{o}=0$ is suitable. There are also further small technical modifications, but none of them is substantial.

\section{Nilpotent and semilinear GPT}

Definition 2.1. A GPT $G$ will be called nilpotent if $G(x, y)=$ o for all but finitely many pairs $(x, y) \in \mathbf{D}$. An algebra $\mathcal{A}=(\mathbf{A} ; *, \mathrm{o})$ will be called nilpotent if for every $w \in \mathbf{A}^{+}, \operatorname{GPT}(\mathcal{A}, w)$ is nilpotent.

To avoid possible misunderstanding, notice that all configurations are usually considered in the definition of nilpotent cellular automata; the above definition deals with finite configurations (i.e., those with finite supports).

DEFINITION 2.2. A language $L$ will be called a simple linear language of degree at most $k$ if there are words $u_{0}, v_{1}, u_{1}, v_{2}, \ldots, u_{k-1}, v_{k}, u_{k}$ such that

$$
L=\left\{u_{0} v_{1}^{i} u_{1} v_{2}^{i} u_{2} \ldots u_{k-1} v_{k}^{i} u_{k}: i>0\right\} .
$$

A language $L$ will be called a simple semilinear language (abbreviated: SSL language) of degree at most $k$ if $L$ is a disjoint union of finitely many simple linear languages of degree at most $k$.

$L$ will be called an SSL language of degree $k$ if L is an SSL language of degree at most $k$ but it is not an SSL language of degree at most $k-1$. $L$ will be called an SSL language if it is an SSL language of degree at most $k$ for some $k \in \mathbf{N}$.

A GPT $G$ will be called an SSL GPT (of degree [at most] $k$ ) if the set of its rows is an SSL language (of degree [at most] $k$ ).

An algebra $\mathcal{A}=(\mathbf{A} ; *, \mathrm{o})$ will be called an $S S L$ algebra (of degree at most $k$ ) if for very $w \in \mathbf{A}^{+}, \operatorname{GPT}(\mathcal{A}, w)$ is $\mathrm{SSL}$ (of degree at most $k$ ). It will be called an $S S L$ algebra of degree $k$ if it is of degree at most $k$ but it is not of degree at most $k-1$.

Notice that an SSL algebra need not be SSL of a (finite) degree because the degrees of its GPT can be arbitrarily large. Every nilpotent algebra is an SSL algebra of degree 1 but the converse does not hold.

Some of the above defined properties can be characterized as follows. $G=$ $\operatorname{GPT}(\mathcal{A}, w)$ is an SSL GPT if and only if for every $c \in \mathbf{A}$ the set $G^{-1}\{c\}$ is 
definable in Presburger arithmetic. $G$ is an SSL GPT of degree at most 2 if and only if the set of its rows is a context-free language.

3. Simple semilinear GPT and the $3 x+1$ problem. For every $y \in \mathbf{N}$ define

$$
T(y)=\frac{3 y+1}{2} \quad \text { if } y \text { is odd, } \quad T(y)=\frac{y}{2} \quad \text { if } y \text { is even. }
$$

Further, write $T^{0}(y)=y$ and $T^{n+1}(y)=T\left(T^{n}(y)\right)$ for every $n, y \in \mathbf{N}$. The sequence $\left(T^{0}(y), T^{1}(y), T^{2}(y), \ldots\right)$ will be called the $T$-trajectory of $y$. There are several unsolved hypotheses connected with the iterations of $T$; for example:

$3 x+1$ CONJECTURE. For every positive integer $y$ there is $n$ such that $T^{n}(y)=1$.

Divergent trajeCtory CONJECTURE (ON $\mathbf{N}$ ). There is no $y \in \mathbf{N}$ such that $\lim _{n \rightarrow \infty} T^{n}(y)=\infty$.

Finite CyCles CONJECTURE (ON $\mathbf{N}$ ). There are only finitely many $y \in \mathbf{N}$ such that there is $n>0$ such that $T^{n}(y)=y$.

Of course, the first hypothesis (called also the Syracuse problem, CollatzKakutani problem, etc.) implies the second and the third ones. All three hypotheses seem to be very hard. For references and history see [18] (where, however, the second and the third hypotheses are formulated for the set $\mathbf{Z}$ of all integers instead of $\mathbf{N}$ ).

Here some algebras (consisting of 7 or 8 elements) will be constructed and some structural questions about their GPT will be shown equivalent to the above hypotheses. So we can conclude that these questions about GPT are also hard. On the other hand, GPT can help us to visualize some results concerning the $3 x+1$ problem.

Definition 3.1. Let $\mathcal{A}_{1}=\left(\mathbf{A}_{1} ; *, \mathrm{o}\right)$, where $\mathbf{A}_{1}=\{\mathrm{o}, 0,1,2,3,4,5\}$ and the operation $*$ is defined by

$$
x * y= \begin{cases}(3 x) \text { MOD } 6+(3 y) \text { DIV } 6 & \text { if } x \neq \mathrm{o}, y \neq \mathrm{o}, \\ \mathrm{o} & \text { if } x=\mathrm{o}, y \in\{\mathrm{o}, 0,1\}, \\ (3 y) \text { DIV } 6 & \text { if } x=\mathrm{o}, y \in\{2,3,4,5\}, \\ 4 & \text { if } x \in\{1,3,5\}, y=\mathrm{o}, \\ \mathrm{o} & \text { if } x \in\{0,2,4\}, y=\mathrm{o} .\end{cases}
$$

The algebra $\mathcal{A}_{1}$ and one of its GPT are displayed in Figure 1. Assume that the substantial part of a row of a $\operatorname{GPT}\left(\mathcal{A}_{1}, w\right)$ does not contain any o. (This assumption is not satisfied in Figure 1; however, any maximal segment of a row not containing any o can be considered as well.) If we understand o as a blank then the way how a row of a $\operatorname{GPT}\left(\mathcal{A}_{1}, w\right)$ is formed from the previous row resembles multiplying by 3 in the number system with base 6 . The difference is at the right end, where:

either 4 arises instead of 3 (so $3 u+1$ is obtained instead of $3 u$ ), or the rightmost zero is lost (so $3 u$ is divided by 6 and $\frac{1}{2} u$ is obtained). 


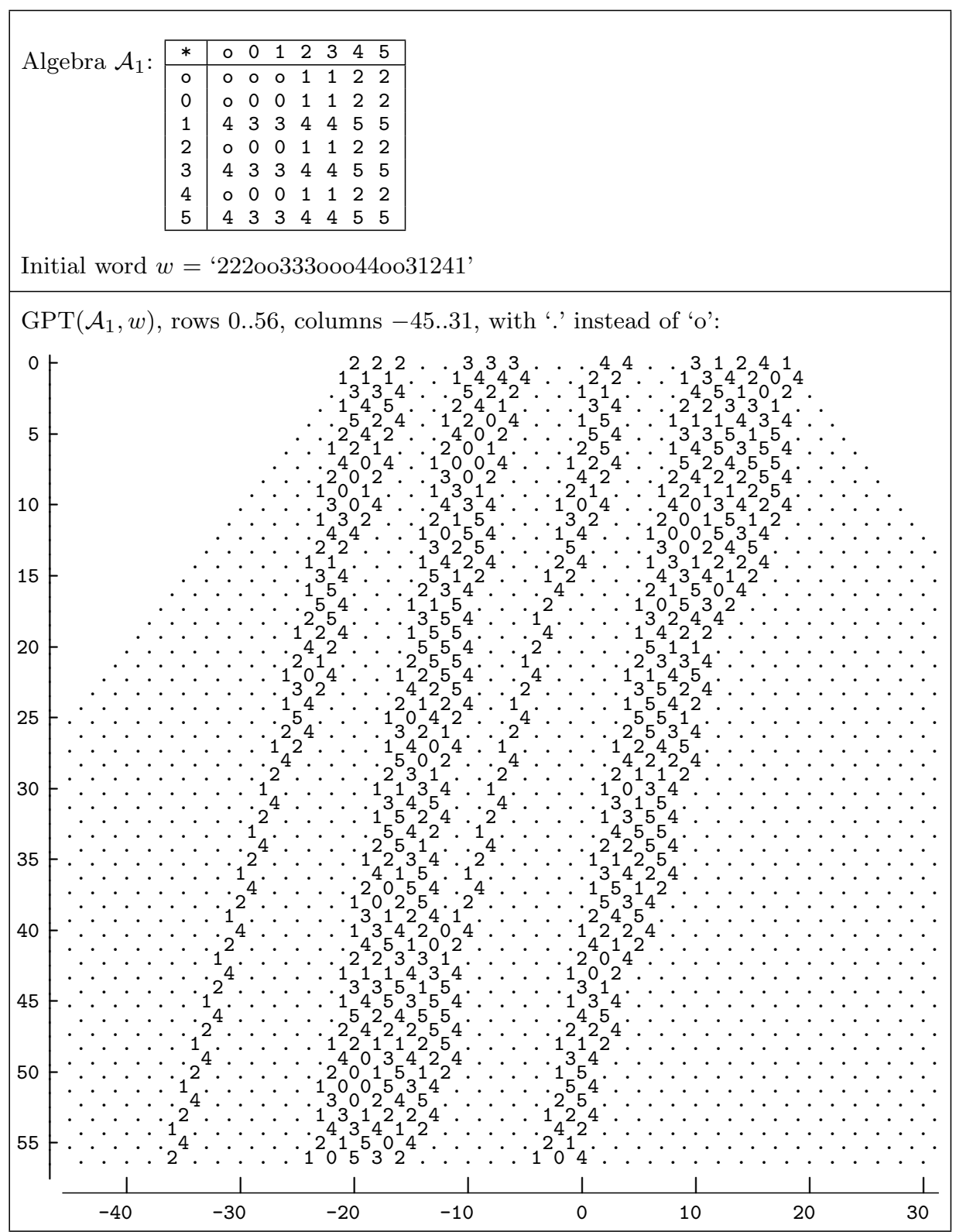

Fig. 1

For every $w \in\left(\mathbf{A}_{1}-\{\mathrm{o}\}\right)^{+}$denote by $\operatorname{val}_{6}(w)$ the integer represented by $w$ in the number system with base 6 (leading zeros are allowed). Then

Lemma 3.2. Let $w \in(\mathbf{A}-\{\mathrm{o}\})^{+}, w(0) \neq 0$ and $q=\liminf _{n \rightarrow \infty} T^{n}\left(\operatorname{val}_{6}(w)\right)$.

$$
\|w\|=\lim _{t \rightarrow \infty} \frac{\operatorname{lmarg}(\mathcal{A}, w ; t)}{t}
$$


exists and

(i) $\|w\|=1 / 3$ if $q=1$;

(ii) $\log _{6} 2-0.072 / q<\|w\|<\log _{6} 2$ if $1<q<\infty$;

(iii) $\|w\|=\log _{6} 2$ if $q=\infty$.

Lemma 3.3. For every $w \in\left(\mathbf{A}_{1}-\{\mathrm{o}\}\right)^{+}$and $c, d \in \mathbf{N}, \operatorname{GPT}\left(\mathcal{A}_{1}, o^{c} w \mathrm{o}^{d}\right)$ is simple semilinear if and only if the T-trajectory of $\operatorname{val}_{6}(w)$ is ultimately periodic.

THEOREM 3.4. The algebra $\mathcal{A}_{1}$ is an SSL algebra of degree 2 if and only if the $3 x+1$ conjecture holds.

The algebra $\mathcal{A}_{1}$ is an SSL algebra if and only if the divergent trajectory conjecture ( on $\mathbf{N}$ ) holds.

The algebra $\mathcal{A}_{1}$ is an $S S L$ algebra of a finite degree if and only if both the divergent trajectory conjecture (on $\mathbf{N}$ ) and the finite cycles conjecture (on $\mathbf{N}$ ) hold.

Remark 3.5. Since the $3 x+1$ conjecture was verified up to $2^{40}$, Lemma 3.2 leaves only an interval of length less than $10^{-13}$ for possible values of $\|w\|$ distinct from $\frac{1}{3}$ and 1. ( $\|w\|=1$ corresponds to some $w$ not considered in Lemma 3.2.)

The nilpotency of GPT is a simpler and more transparent property than their simple semilinearity. Therefore it would be nice to replace the SSL algebra in Theorem 3.4 by a nilpotent algebra. We shall do it for the first part of Theorem 3.4 , but the cardinality of the algebra will increase to 8 .

Definition 3.6. Let $\mathcal{A}_{2}=\left(\mathbf{A}_{2} ; \oplus, \mathrm{o}\right)$, where $\mathbf{A}_{2}=\{\mathrm{o}, 0,1,2,3,4,5, \mathrm{I}\}$ and the operation $\oplus$ is defined by

$$
x \oplus y= \begin{cases}\mathrm{o} & \text { if } x=\mathrm{I}, y=\mathrm{o}, \\ \mathrm{I} & \text { if } x=\mathrm{o}, y=2, \\ x^{\prime} * y^{\prime} & \text { otherwise, }\end{cases}
$$

where $x^{\prime}=x$ if $x \neq \mathrm{I}$ and $\mathrm{I}^{\prime}=1$.

The algebra $\mathcal{A}_{2}$ and one of its GPT are displayed in Figure 2. If we compare this with Figure 1, the main difference is that periodic "tails" (consisting of 4,2,1) have been cut off.

Lemma 3.7. For every $w \in\left(\mathbf{A}_{1}-\{\mathrm{o}\}\right)^{+}, \operatorname{GPT}\left(\mathcal{A}_{2}, w\right)$ is nilpotent if and only if there is $n$ such that $T^{n}\left(\operatorname{val}_{6}(w)\right)=1$.

THEOREM 3.8. The algebra $\mathcal{A}_{2}$ is nilpotent if and only if the $3 x+1$ conjecture holds.

Just as 1 was split into two elements 1 , I in the above construction of $\mathcal{A}_{2}$, also the other elements of the periodic tails can be used. We shall formulate the result for the element 4 .

Theorem 3.9. Let $\mathcal{A}_{3}=\left(\mathbf{A}_{3} ; \otimes, \mathrm{o}\right)$, where $\mathbf{A}_{3}=\{\mathrm{o}, 0,1,2,3,4,5, \mathrm{~F}\}$ and the operation $\otimes$ is defined by 


$$
x \otimes y= \begin{cases}\mathrm{o} & \text { if } x=\mathrm{o}, y=\mathrm{F}, \\ \mathrm{F} & \text { if } x=1, y=\mathrm{o}, \\ x^{\prime} * y^{\prime} & \text { otherwise, }\end{cases}
$$

where $x^{\prime}=x$ if $x \neq \mathrm{F}$ and $\mathrm{F}^{\prime}=4$. Then the algebra $\mathcal{A}_{3}$ is nilpotent if and only if the $3 x+1$ conjecture holds.

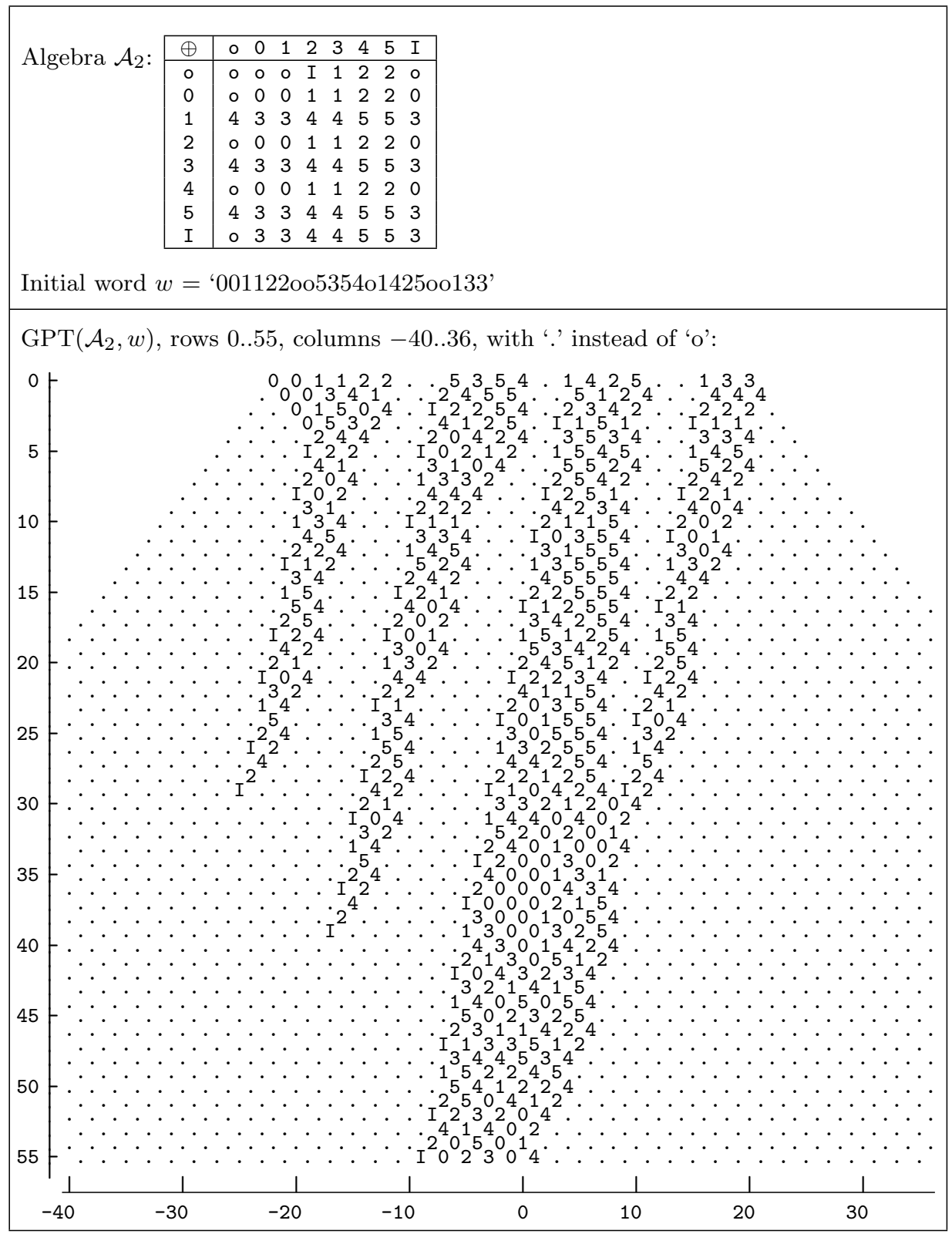

Fig. 2 
4. Speeds of configuration growth in GPT - Definition. Generalized Pascal triangles can be considered as computations of one-dimensional cellular automata from finite initial configurations. (The quieting state will be o or an $n$-tuple consisting of o, where $n$ is determined by the type of neighbourhood.) Cellular automata are often used to simulate some physical processes. Imagine for example waves spreading on a water surface (in a narrow and very long reservoir for the one-dimensional case). If we throw several stones into water the waves will spread on the water surface. The size and the form of the waves will depend on the number of stones thrown, their weights etc., but the speed of the wave head will usually be independent of these circumstances. Hence it is natural to require that a cellular automaton used to simulate these waves has the following property:

In every computation with finite nonempty initial configuration, the length of the support is (asymptotically) a linear function of time, and the speed of its growth does not depend on the initial configuration.

These considerations motivate the following definition:

Definition 4.1. Let $\mathcal{A}=(\mathbf{A} ; *, \mathrm{o})$ be a finite algebra and $\alpha$ be a real.

(i) We shall say that $\alpha$ is the speed of configuration growth of $\mathcal{A}$, and write $\operatorname{SpGr}(\mathcal{A})=\alpha$, if there is a constant $c$ such that for every $w \in \mathbf{A}^{+}-\{\mathrm{o}\}^{+}$and every $t \in \mathbf{N}$

$$
|| \operatorname{SP}(\mathcal{A}, w ; t)|-(|\mathrm{SP}(\mathcal{A}, w ; 0)|+\alpha t)| \leq c .
$$

(ii) We shall write $\operatorname{SpGr}_{1}(\mathcal{A})=\alpha$ if for every $w \in \mathbf{A}^{+}-\{\mathrm{o}\}^{+}$there is a constant $c$ such that (4.1.1) holds.

If $\operatorname{SpGr}(\mathcal{A})$ is defined then $\operatorname{SpGr}_{1}(\mathcal{A})$ is also defined and $\operatorname{SpGr}_{1}(\mathcal{A})=\operatorname{SpGr}(\mathcal{A})$. The converse is not true: it may happen that $\operatorname{SpGr}_{1}(\mathcal{A})$ is defined and $\operatorname{SpGr}(\mathcal{A})$ does not exist. Analogously, it may happen that

$$
\lim _{t \rightarrow \infty} \frac{|\operatorname{SP}(\mathcal{A}, w ; t)|}{t}
$$

exists for every $w \in \mathbf{A}^{+}-\{\mathrm{o}\}^{+}$and does not depend on $w$, and, despite this, $\operatorname{SpGr}_{1}(\mathcal{A})$ does not exist. Hence the condition (i) in Definition 4.1 is rather strong. It requires that the configuration growth is "as linear as possible" and simultaneously "as uniform as possible". This makes existence results about SpGr also rather strong, e.g. stronger than those about $\mathrm{SpGr}_{1}$. In the next section it is shown how to meet this condition in a nontrivial way.

5. Irrational speeds of configuration growth. It is very easy to find an algebra $\mathcal{A}$ such that $\operatorname{SpGr}(\mathcal{A})=1$; also lower rational speeds can easily be obtained. On the other hand, by the simulation of a suitable Turing machine also irrational speeds can be obtained; however, in this case the speed of growth usually depends on the initial configuration (and it does not necessarily exist for all initial configurations). So the conditions of Definition 4.1 need not be satisfied. 
We shall present a method to obtain irrational speeds based on a numbertheoretical idea. For this purpose, we shall generalize the notion of positional number system and introduce an auxiliary set $\mathbf{K}$.

Definition 5.1. (i) A positional number system is an ordered triple

$$
(\mathbf{A}, \mathrm{val}, b) \text {, }
$$

where $\mathbf{A}$ is an alphabet (of digits), val is a mapping of $\mathbf{A}$ into the set of nonnegative reals and the real $b>1$ is the basis.

(ii) The function val is extended to the set $\mathbf{A}^{+}$by the formula

$$
\operatorname{val}\left(u_{n} u_{n-1} \ldots u_{1} u_{0}\right)=\sum_{i=0}^{n} \operatorname{val}\left(u_{i}\right) \cdot b^{i}
$$

for every $n \in \mathbf{N}$ and all $u_{n}, u_{n-1}, \ldots, u_{1}, u_{0} \in \mathbf{A}$.

Generally speaking, the above definition gives neither the existence nor the uniqueness of representations of nonnegative reals; we do not investigate any details not concerning our main goal.

Definition 5.2. $\quad \mathbf{K}$ is the set of all reals $\alpha$ for which there are positive reals $\gamma_{1}, \gamma_{2}, \ldots, \gamma_{k}$ and nonnegative integers $c_{i}, d_{i j}(i, j \in\{1,2, \ldots, k\})$ such that

$$
\begin{gathered}
\alpha=c_{1} \gamma_{1}+c_{2} \gamma_{2}+\ldots+c_{k} \gamma_{k} \quad \text { and } \\
\left(\begin{array}{c}
\gamma_{1}^{2} \\
\gamma_{2}^{2} \\
\vdots \\
\gamma_{k}^{2}
\end{array}\right)=\left(\begin{array}{cccc}
d_{11} & d_{12} & \ldots & d_{1 k} \\
d_{21} & d_{22} & \ldots & d_{2 k} \\
\vdots & \vdots & & \vdots \\
d_{k 1} & d_{k 2} & \ldots & d_{k k}
\end{array}\right) \cdot\left(\begin{array}{c}
\gamma_{1} \\
\gamma_{2} \\
\vdots \\
\gamma_{k}
\end{array}\right)
\end{gathered}
$$

The parameters in the main theorem of this section (Theorem 5.4) will run over $\mathbf{K}$. Therefore the following theorem is presented which shows how rich (items $\mathrm{a}, \mathrm{b}, \mathrm{c}$ ) and how poor (items $\mathrm{d}$, e) the set $\mathbf{K}$ is:

TheOREM 5.3. a) The set $\mathbf{K}$ contains all nonnegative integers.

b) The set $\mathbf{K}$ is closed under addition, multiplication and taking $k$-th roots $(k=2,3,4, \ldots)$.

c) If $\alpha_{0}, \alpha_{1}, \ldots, \alpha_{n-1} \in \mathbf{K}$ and $\gamma$ is a positive real such that

$$
\gamma^{n}=\alpha_{n-1} \gamma^{n-1}+\ldots+\alpha_{1} \gamma+\alpha_{0}
$$

then $\gamma \in \mathbf{K}$.

d) The set $\mathbf{K}$ contains no negative real and no real $x$ such that $0<x<1$.

e) All elements of $\mathbf{K}$ are algebraic integers.

Theorem 5.4. If $\alpha, \beta \in \mathbf{K}, b \in \mathbf{N}$ and $0<\alpha . \beta<b$ then there is a finite algebra $\mathcal{A}=(\mathbf{A} ; *, \mathrm{o})$ such that

$$
\operatorname{SpGr}(\mathcal{A})=\frac{\log \alpha}{\log b-\log \beta} .
$$


Id ea of proof. Substantial parts of rows of $\operatorname{GPT}(\mathcal{A}, w)$ are understood as numbers written in a positional number system with the base $\delta=b / \beta$, and forming a new row will correspond to multiplication by $\alpha$. This adds $\log _{\delta} \alpha$ to the length of the substantial part on the average. The positional number system must be chosen so that multiplying by $\alpha$ is possible without "long carries". (They occur in the usual decimal system e.g. for $\alpha=3$ but do not occur for $\alpha=2,5$.) Moreover, $\mathrm{o}=0$ in the constructed algebra, and we must arrange that no new zeros arise at the ends of substantial parts.

\section{References}

[1] B. A. Bondarenko, Generalized Pascal triangles and pyramids, their fractals, graphs and applications, Fan, Tashkent 1990 (in Russian).

[2] A. Černý and J. Gruska, Modular trellises, in: G. Rozenberg and A. Salomaa (eds.), The Book of L, Springer, Berlin 1985, 45-61.

[3] —, - Modular real-time trellis automata, Fund. Inform. 11 (3) (1986), 253-282.

[4] K. Culik II, J. Gruska and A. Salomaa, Systolic trellis automata. Part I, Internat. J. Comput. Math. 15 (1984), 195-212.

[5] - - - - Systolic trellis automata. Part II, ibid. 16 (1985), 3-22.

[6] K. Culik II, L. P. Hurd and S. Y u, Computation theoretic aspects of cellular automata, Physica D 45 (1990), 357-378.

[7] J. Gruska, Systolic automata-power, characterizations, nonhomogeneity, in: Proc. Math. Foundations of Computer Science, Praha, Lecture Notes in Comput. Sci. 176 Springer, Berlin 1984, 32-49.

[8] —, Systolic architectures, systems and computations, in: T. Lepistö and A. Salomaa (eds.), Automata, Languages and Programming, Proc. ICALP, Tampere, Lecture Notes in Comput. Sci. 317, Springer, Berlin 1988, 254-270.

[9] —, Synthesis, structure and power of systolic computations, Theoret. Comput. Sci. 71 (1988), 47-77.

[10] I. Korec, Generalized Pascal triangles, D.Sc. thesis, UK Bratislava, 1984 (in Slovak).

[11] - Generalized Pascal triangles. Decidability results, Acta Math. Univ. Comenian. 46-47 (1985), 93-130.

[12] - Multiples of an integer in the Pascal triangle, ibid., 83-91.

[13] - Generalized Pascal triangles, in: K. Halkowska and S. Stawski (eds.), Proc. V Universal Algebra Symposium, Turawa, Poland, May 1988, World Scientific, Singapore 1989, 198218.

[14] _, Semilinear real-time systolic trellis automata, in: J. Csirik, J. Demetrovics and F. Gécseg (eds.), Fundamentals of Computation Theory, Proc. FCT '89, Lecture Notes in Comput. Sci. 380, Springer, Berlin 1989, 267-276.

[15] - , Pascal triangles modulo $n$ and modular trellises, Comput. Artificial Intelligence 3 (1990), 105-113.

[16] - Irrational speeds of configuration growths in generalized Pascal triangles, Theoret. Comput. Sci., to appear.

[17] —, The $3 x+1$ problem, generalized Pascal triangles and cellular automata, Math. Slovaca 42 (5) (1992), 547-563.

[18] J. C. Lagarias, The $3 x+1$ problem and its generalizations, Amer. Math. Monthly 92 (1985), 3-23. 